\title{
Teoria das Molduras Relacionais e Desfusão Cognitiva: Discussões e direções para a pesquisa experimental
}

\section{Relational Frame Theory and Cognitive Defusion: Discussions and directions for experimental research}

\section{Teoria de los Marcos Relacionales y Defusion Cognitiva: Discussiones y direcciones para la investigación experimental}

\author{
Matheus H. S. Mello', Paola E. M. Almeida ${ }^{1}$
}

[1] Pontifícia Universidade Católica, São Paulo I Título abreviado: Estudo experimental da desfusão cognitiva I Endereço para correspondência: Matheus H. S. Mello, Laboratório de Psicologia Experimental: Análise do Comportamento, Rua Bartira, 387, CEP 05009-000 - São Paulo - SP I Email: matheushsmello@gmail. com I doi: 10.18761/PAC.2021.v12.RFT.15

Resumo: A Teoria das Moldura Relacionais (RFT) é uma abordagem analítico-comportamental para o estudo do comportamento verbal. A Terapia da Aceitação e Compromisso (ACT) é uma abordagem terapêutica filosoficamente fundamentada no contextualismo funcional, e conceitualmente influenciada pela RFT e por princípios comportamentais básicos. Apesar de dizer-se baseada na RFT, muitos conceitos e termos da ACT carecem de definições e validação experimental conceitualmente consistentes. A ACT e a RFT fazem parte de um movimento ainda maior, denominado Ciências Comportamentais Contextuais (CBS), que propõe um modelo reticulado para a produção de conhecimento, no qual pesquisa básica e aplicada evoluem independentemente, mas em continua interação. O presente artigo discute os métodos de pesquisa adotada para definir, investigar e aproximar uma das principais técnicas terapêuticas da ACT, nomeada pelo termo médio desfusão cognitiva, dos processos comportamentais básicos. Os problemas práticos e experimentais decorrentes destas estratégias de investigação são analisados criticamente. Por fim, propõe-se novas direções e estratégias experimentais para a investigação e definição da desfusão cognitiva.

Palavras-chave: Teoria das Molduras Relacionais; Terapia da Aceitação e Compromisso; Desfusão Cognitiva; Análise Experimental do Comportamento 
Abstract: Relational Frames Theory (RFT) is a behavioral-analytic approach for the study of verbal behavior. Acceptance and Commitment Therapy (ACT) is a therapeutic philosophically based on functional contextualism, and conceptually influenced by RFT and basic behavioral principles. Despite it said to be based on the RFT, many ACT concepts and terms lack conceptually consistent definitions and experimental validation. ACT and RFT are part of an even larger movement, named Contextual Behavioral Sciences (CBS), which proposes a reticulated model to produce knowledge, in which basic and applied research evolve independently, but in continuous interaction. This article discusses the research methods adopted to define, investigate and approximate one of the main therapeutic techniques of ACT, named by the middle-level term cognitive defusion, of the basic behavioral processes. The practical and experimental problems arising from these strategies of investigation are analyzed critically. Finally, new directions and experimental strategies are proposed for the investigation and definition of cognitive defusion.

Keywords: Relational Frame Theory; Acceptance and Commitment Therapy; Cognitive Defusion; Experimental Analysis of Behavior

Resumen: La Teoría de los Marcos Relacionales (RFT) es un enfoque analítico-comportamental al estudio del comportamiento verbal. La Terapia de Aceptación y Compromiso (ACT) es un abordaje terapéutico basado filósoficamente en el contextualismo funcional y conceptualmente influenciado en RFT y principios básicos de comportamiento. Aunque se disse que se basa en la RFT, muchos conceptos y términos de la ACT carecen de definiciones y validación experimental consistentes conceptualmente. La ACT y la RFT hacen parte de un movimiento aún más grande, la llamada Ciencia Conductual Contextual (CBS), que propone un modelo reticulado para la producción de conocimiento, en la cual investigación básica y aplicada evolucionan de modo independiente, pero en una interacción continua. El presente artículo discute los métodos de investigación adoptados para definir, investigar y aproximar una de las principales técnicas terapéuticas del ACT denominada por el término medio defusión cognitiva, a los procesos comportamentales básicos. Los problemas prácticos y experimentales que surgen de estas estrategias de investigación son analizados críticamente. Por último, se proponen nuevas direcciones y estrategias experimentales para la investigación y definición de la defusión cognitiva.

Palabras clave: Teoría de los Marcos Relacionales; Terapia Aceptación y Compromiso; Difusión Cognitiva; Analisis Experimental de la Conducta

O presente artigo foi realizado com total apoio do Conselho Nacional de Desenvolvimento Científico e Tecnológico (CNPq). 
A Teoria das Molduras Relacionais (Relational Frame Theory - RFT) é uma proposta comportamental contemporânea para o estudo do comportamento verbal (Hayes, Barnes-Holmes \& Roche, 2001). Para a RFT, os fenômenos comumente descritos como linguagem e cognição abrangem eventos comportamentais que podem ser explicados a partir do conceito de Responder Relacional Arbitrariamente Aplicável (RRAA), um operante generalizado contextualmente controlado. Em virtude da complexidade e densidade desta proposta teórica, cuja compreensão é imprescindível para as discussões levantadas no presente artigo, é importante deixar claro os princípios fundamentais da RFT.

O termo responder relacional diz respeito a um comportamento operante sob controle de relações entre eventos ambientais. Para que este tipo de controle se estabeleça, é necessário um Treino de Múltiplos Exemplares, que envolve o reforçamento de respostas à propriedades relacionais específicas, presentes na relação entre diferentes conjuntos de estímulos. Por exemplo, é possível reforçar a resposta de uma criança de apontar ao maior de diferentes pares de objetos sempre que um adulto demanda que escolha o maior. Após um histórico de reforçamento consistente desse tipo, a criança pode apontar para o maior de um conjunto completamente novo de objetos assim que solicitado, indicando que o seu responder ficou sob controle da relação "maior que" - uma propriedade que não existe isoladamente, mas apenas enquanto parte de relações entre eventos.

O comportamento exemplificado acima ainda não é o foco da RFT, pois trata-se um responder relacional não-arbitrário, uma vez que os antecedentes controladores consistem em relações formais entre estímulo. Dada a exposição às contingências adequadas, respostas relacionais podem ficar sob controle discriminativo de outras variáveis ambientais presentes ao longo da história de reforçamento, cunhadas de dicas contextuais, de tal forma que a sua ocorrência deixa de depender unicamente das propriedades formais de conjuntos de estímulos. No exemplo anterior, maior que pode adquirir controle contextual sobre o responder à esse tipo de relação de comparação. Uma vez estabelecido o controle contextual, as dicas contextuais podem ser utilizadas para se estabelecer relações arbitrárias, que podem ser aplicadas a quaisquer conjuntos de eventos, mesmo aqueles que não apresentam as propriedades físicas definidoras da relação (Hayes et al., 2001). No presente caso, os diferentes tamanhos de objetos. É nesse sentido que o responder relacional se torna arbitrariamente aplicável (Hayes et al., 2001; Perez, Nico, Kovac, Fidalgo \& Leonardi, 2013). Por exemplo, ao assistir um filme de heróis em que os personagens apresentam a mesma estatura, um pai diz ao filho que "o batman é maior do que o super-homem". Posteriormente, ao ser questionado qual é o maior entre ambos os heróis, a criança pode prontamente se orientar para batman. Aqui, a dica maior que foi empregada arbitrariamente pelo pai com base em convenções sociais, visto que os personagens não apresentam características definidoras deste tipo de relação; para a criança, esta dica contextual foi a variável ambiental responsável por controlar o estabelecimento, no seu repertório, de uma relação de comparação arbitrária entre ambos os personagens. Em outras palavras, o padrão de responder relacional contextualmente controlado de comparação foi arbitrariamente aplicado à um conjunto de estímulos, independente das suas características formais.

Segundo Hayes et al. (2001), a comunidade verbal sustenta contingências que consistentemente evocam e fortalecem respostas relacionais arbitrárias tanto unidirecionais (e.g. palavra $\rightarrow$ objeto) quanto bidirecionais (e.g. objeto $\rightarrow$ palavra) aos mais diversos eventos ambientais. Em virtude do treino direto deste responder bidirecional à inúmeros estímulos, respostas relacionais bidirecionais podem ser derivadas, sem reforçamento explicito, a partir do treino de respostas unidirecionais à novos conjuntos de estímulos. O produto desse histórico de reforçamento não é apenas o fortalecimento de instâncias específicas de respostas relacionais, mas sim do operante generalizado de responder a relações arbitrárias entre eventos (RRAA) sem treino explícito (Hayes et al., 2001; Perez et al., 2013).

O RRAA é definido pelas propriedades contextualmente controladas de (i) implicação mútua (se A está relacionado com $\mathrm{B}$, implica-se que $\mathrm{B}$ está mutuamente relacionado com A), (ii) implicação combinatória (as relações mútuas A-B e B-C podem ser combinadas, implicando uma terceira relação mútua $\mathrm{A}-\mathrm{C}$ ), e (iii) transformação de fun- 
ção (as funções comportamentais de estímulos são modificadas de acordo com as relações das quais participam). No que diz respeito à sua regulação ambiental, o RRAA é sensível a duas formas de controle antecedente, descritas como contexto relacional e contexto funcional. Por contexto relacional entende-se as variáveis controladoras do tipo de relação estabelecida entre eventos; por exemplo, em "Sócrates é maior que Platão", a palavra maior que controla o estabelecimento de uma relação de comparação arbitrárias entre Sócrates e Platão. Por outro lado, o contexto funcional se refere ao controle contextual sobre a transformação de função entre estímulos. Por exemplo, em "imagine a temperatura de sorvete", a palavra sorvete está relacionada ao alimento em si, mas não compartilha de todas as suas funções evocativas - e.g. a resposta de morder ocorre frente ao alimento, mas não à palavra. $\mathrm{O}$ enunciado imagine a temperatura seria um contexto responsável por selecionar a transformação das funções táteis do alimento para a palavra sensação térmica, e não outras (Hayes et al., 2001; Hayes, Strosahl \& Wilson, 2012a ). Nota-se assim que a relação estabelecida entre estímulos depende do tipo de contexto relacional, enquanto as funções transformadas a partir destas relações depende do contexto funcional em vigor.

O termo "Moldura Relacional" foi empregado para descrever padrões específicos deste operante generalizado pois, metaforicamente, assim como uma moldura pode ser aplicada a diferentes pinturas, uma resposta relacional sob controle contextual pode ser aplicada a diferentes conjuntos de estímulos. Molduras Relacionais, portanto, são as diferentes classes de respostas relacionais arbitrárias, definidas pelas variáveis contextuais que as controlam. Estas classes envolvem relações de coordenação (e.g. igual a), oposição (e.g. contrário de), distinção (e.g. diferente de), comparação (e.g. maior que), hierárquicas (e.g. parte de), temporalidade (e.g. antes de), espacialidade (e.g. acima de), condicionalidade (e.g. Se...Então), e relações dêiticas (e.g. Aqui-Lá) entre estímulos (Hayes et al., 2001).

Em resumo, o RRAA é um operante generalizado, enquanto molduras relacionais são os padrões específicos deste operante. Acrescenta-se ainda que, conforme a proposta da RFT, o conceito de comportamento verbal passa a ser definido como o com- portamento de "emoldurar estímulos relacionalmente" (Hayes et al., 2001, p. 45, tradução nossa). No mesmo sentido, estímulos verbais são entendidos como estímulos cujas funções comportamentais foram derivadas em virtude da sua participação em molduras relacionais (Hayes et al., 2001).

A RFT permite, assim, explicar de que maneira eventos ambientais adquirem indiretamente funções comportamentais, independente de exposição direta às contingências operantes ou respondentes: enquanto contextos relacionais podem controlar o estabelecimento e derivação de novas relações mútuas e combinatórias entre estímulos, contextos funcionais podem controlar a aquisição de diferentes funções por estes estímulos, em conformidade com a natureza das relações que participam (Blackledge \& Drake, 2013; Hayes et al, 2001; Hayes et al., 2012a). A RFT é, portanto, um instrumento teórico promissor para a investigação de aspectos singulares do ser humano, principalmente daqueles envolvidos no comportamento verbal, como a possibilidade de responder à estímulos sem nunca tê-los experienciados anteriormente, o fenômeno da insensibilidade às contingências de reforçamento e o treino necessário para que o ouvinte compreenda e responda ao comportamento do falante (Hayes et al., 2001).

Sob influência da RFT, a Terapia da Aceitação e Compromisso (Acceptance and Commitment Therapy - ACT) concebe que o sofrimento psicológico tem como uma das principais fontes o controle do comportamento por funções de estímulos derivadas (Blackledge \& Drake, 2013; Hayes et al., 2012a). Conforme a concepção de psicopatologia da ACT, a função aversiva de muitos estímulos, públicos e privados, pode ter sido adquirida não só via experiência direta, mas também derivada de relações complexas e extensas, estabelecidas verbalmente com outros estímulos. As funções de estímulo aversivas derivadas poderiam sobrepor outras fontes de controle ambiental, fortalecendo repertórios de esquiva generalizadas - que, por sua vez, implicaria no distanciamento de reforçadores positivos importantes ao indivíduo, potencializando o sofrimento individual. Nesse sentido, o modelo de tratamento adotado pela ACT envolve, de maneira geral, intervenções direcionadas à (i) reduzir o controle problemático por funções derivadas 
sobre o comportamento, (ii) fortalecer o controle por regras que descrevem reforçadores relevantes ao cliente, e (iii) construir repertórios consistentes com estas regras (Blackledge \& Drake, 2013; Hayes et al., 2012a). Conforme o modelo atual da ACT, suas intervenções são divididas em seis componentes: aceitação, desfusão cognitiva, contato com o momento presente, self-como-contexto, valores, e ações comprometidas (Blackledge e Drake, 2013; Hayes et al., 2012a).

\section{RFT e Ciências Comportamentais Contextuais: Propostas e desafios para o desenvolvimento da área}

Além das relações teóricas e práticas discutidas, a RFT e a ACT integram um campo da ciência ainda maior, denominado Ciência Comportamental Contextual (Contextual Behavioral Science - CBS). A CBS define-se como uma estratégia para o desenvolvimento científico e prático das ciências comportamentais, tendo como fundamento filosófico o contextualismo funcional (Hayes, Barnes-Holmes, \& Wilson, 2012b; Levin, Twohig, \& Smith, 2016 ).

Apesar das influências iniciais do behaviorismo radical, os proponentes da CBS argumentam que o movimento possui características próprias, como a grande ênfase no estudo de processos verbais, pesquisa básica com participante humanos e visão contextualista do comportamento. Essas características, argumentam seus proponentes, seriam diferentes às da Análise do Comportamento - o suficiente para justificar uma cisão e a constituição de uma ciência independente (Hayes et al., 2012b).

Tal separação envolveria ainda a adoção de uma forma de interação diferente entre os domínios de pesquisa básica e aplicada descritos como estratégias top-down e bottom-up. As estratégias bottom-up permitem o desenvolvimento de conceitos mais precisos e com maior grau de rigor conceitual construído a partir de dados resultantes da pesquisa básica; por outro lado, a sua evolução é lenta e muitas vezes distante de fenômenos clinicamente relevantes. Já a estratégia top-down envolve uma maior ênfase em fenômenos complexos, permitindo o desenvolvimento de tecnologias e conceitos para lidar com as demandas clínicas; entretanto, devido à distância da pesquisa básica e de técnicas de investigação precisas, está mais sujeito à adoção de explicações confusas e imprecisa, e cuja efetividade ainda merece ser testada (Barnes-Holmes, Hussey, McEnteggart, Barnes-Holmes \& Foody, 2016; Hayes et al., 2012b). Como uma alternativa a ambas as estratégias, a CBS propõe a adoção do denominado modelo reticulado - o qual, por sua vez, encoraja tanto a continua produção no domínio aplicado, independente da ausência de respaldo conceitual e empírico para tecnologias desenvolvidas, quanto no domínio básico, independente de utilidade aplicada imediata. Concomitantemente ao desenvolvimento individual, encoraja-se esforços na integração e aprimoramento mútuo entre ambos os domínios (Barnes-Holmes et al., 2016; Levin et al., 2016).

Adicionalmente, a ciência proposta pela CBS evolve a adoção dos chamados termos médios (e.g. fusão cognitiva, aceitação, self-conceitual): termos com (i) um menor grau de precisão, (ii) baseados em princípios básicos, que descrevem (iii) conjuntos de relações funcionais, e que (iv) orientam a aplicação de técnicas específicas (Hayes et al., 2012b). Todos os componentes da ACT são descritos por conceitos desta natureza. A utilização de uma terminologia de nível médio, defendem Hayes et al. (2012b), é uma prática considerada adequada e encorajada pela CBS, pois, além de alinhada à proposta de uma estratégia reticulada, o menor grau de precisão conceitual dos termos médios seria compensado pelo valor pragmático em orientar a prática de terapeutas pouco familiarizados com princípios comportamentais básicos. Os termos médios, portanto, permitiriam que terapeutas apliquem técnicas e produzam mudança de comportamento, mesmo que não compreendam conceitos básicos da ciência que respalda as técnicas que aplicam (Barnes-Holmes et al., 2016; Hayes et al., 2012b; Levin et al., 2016).

Uma vez que a estratégia reticulada encoraja a interação constante entre os diferentes domínios, a utilização de termos médios pela ACT - dimensão aplicada - traria a necessidade investigações experimentais e conceituais direcionadas à refinar a precisão destes termos - dimensão básica. Da mesma forma, desenvolvimentos empíricos da RFT contribuiriam com a criação de novas técnicas clínicas 
e derivação de novos conjuntos de termos médios (Barnes-Holmes et al., 2016; Hayes et al., 2012b; Levin et al., 2016).

Apesar do modelo reticulado incentivar uma interação constante entre os diferentes níveis de análise, a produção científica aos moldes da CBS tem progredido de maneira desigual entre as dimensões básica, conceitual e prática da produção de conhecimento. Alguns autores chamam atenção ao fato de que a crescente produção de dados de eficácia e disseminação dos procedimentos da ACT não foi acompanhada por um progresso semelhante no campo da pesquisa empírica, principalmente no que diz respeito à investigação dos processos básicos envolvidos em comportamentos e intervenções clinicamente relevantes, de tal maneira que muitos dos aspectos abrangidos pelos termos médios ainda exigem o escrutínio experimental e rigor conceitual ideal (Blackledge \& Drake, 2013; Dymond, Roche \& Bennet, 2013; Levin \& Villatte, 2016). Parte deste cenário pode ser atribuído ao fato de que ACT não se constitui como uma derivação direta da RFT: o desenvolvimento de ambos é melhor entendido como um processo de coevolução e influência mútua. Isto é, ao mesmo tempo que ocorriam os refinamentos conceituais e validação experimental dos princípios básicos da RFT, os conceitos da ACT eram ampliados e explicados teoricamente de acordo com a RFT - uma teoria ainda em construção. Com efeito, este processo pode ser entendido como uma forma de reticulação entre prática aplicada e pesquisas teórico-experimentais; como resultado, os numerosos e frequentes termos médios que compõe o vocabulário da ACT ainda se encontram distantes do completo respaldo teórico na RFT.

Ainda além, Barnes-Holmes et al. (2016) consideram problemática a assumida funcionalidade dos termos médios, visto que "os próprios termos não parecem aderir ao critério de verdade filosófica de precisão e influência que orienta o contextualismo funcional.” (p. 366, tradução nossa).

Com efeito, a funcionalidade de um termo está diretamente ligada à sua precisão e à forma de investigação a partir da qual é extraído. $\mathrm{O}$ valor pragmático de um conceito, postulou Skinner (1945/1972), reside na medida em que a sua utilização permite ao cientista proceder efetivamente sobre o fenômeno de interesse. Para que o conceito oriente adequadamente o comportamento do cientista, é necessário que a comunidade verbal defina precisamente à quais fenômenos do mundo este conceito se refere - e tal precisão descritiva pode ser obtida através do rigoroso escrutínio experimental. Afinal, um dos compromissos primordiais da pesquisa básica diz respeito à manipulação de variáveis para a verificação de relações funcionais entre eventos; e, a partir da observação cuidadosa e sistemática dessas relações, é possível atribuir precisamente termos descritivos para as suas dimensões e propriedades.

Nessa direção teórica, o distanciamento - decorrente da estratégia reticulada - entre a utilização de um termo médio e a investigação empírica necessária para esclarecer à quais processos comportamentais o termo se refere implica em imprecisões conceituais. Precisamente, o problema não é o termo médio, mas sim a falta de clareza em relação aos procedimentos, processos, resultados ou relações funcionais aos quais o termo se refere (Assaz, Roche, Kanter \& Oshiro, 2018; Barnes-Holmes et al., 2016). Uma vez que o conceito se torna impreciso, o seu valor pragmático é reduzido: tanto para o pesquisador básico, que encontrará dificuldades em delinear experimentos com base no conceito, quanto para o praticante, que encontrará dificuldades em identificar e manipular as variáveis descritas pelo termo no setting clínico. Visto isso, é de grande relevância teórica e prática que pesquisadores empreendam esforços direcionadas em esclarecer quais são as relações funcionais e procedimentos descritos pelos termos médios. Tais esforços permitiriam modificar, manter ou eliminar completamente tais termos a depender da sua funcionalidade.

Historicamente, a desfusão cognitiva é um dos componentes centrais da ACT e, enquanto termo médio, está sujeita às problemáticas discutidas acima (Blackledge \& Drake, 2013; Zettle, 2011). Através de uma discussão metodológica, o presente artigo tem como objetivo abordar (i) de que maneira pesquisadores têm buscado definir, investigar e aproximar o termo médio desfusão cognitiva e os processos comportamentais básicos relacionados a este conceito, (ii) os problemas práticos e experimentais decorrentes destas formas de investigação, e, por fim, (iii) propor rumos mais adequados para o estudo deste conceito. 


\section{Desfusão Cognitiva: Intepretação e experimentação}

Desde a origem da ACT às investigações recentes, pesquisadores têm se engajado em tentativas de esclarecer quais são os processos comportamentais básicos envolvidos nos procedimentos e fenômenos descritos por termos médios. Em relação à desfusão cognitiva, tal aproximação permitiria elucidar confusões conceituais, a identificar quais são, exatamente, os processos responsáveis pela mudança comportamental promovida por estas estratégias, bem como evitar a atribuição de diversos procedimentos e processos a um mesmo termo (Assaz et al., 2018; Barnes-Holmes et al., 2016; Levin et al., 2016).

Com efeito, identificar os processos comportamentais responsáveis pela efetividade das estratégias referidas pelos termos médios têm sido considerada uma tendência atual pelos próprios proponentes da CBS:

\footnotetext{
"A questão contemporânea para a psicologia clínica não é mais apena se se um tratamento é efetivo, mas sim o que o torna efetivo. Responder o "por quê" se mostrou muito mais desafiador do que responder "o quê", geralmente abordado pelas pesquisas de resultado" (Barnes-Holmes et al. 2016, p. 367, tradução nossa)
}

A buscar por estas respostas parece ser uma preocupação de longa data da tradição comportamental, presente desde os esforços iniciais em se utilizar de teorias da aprendizagem para a construção de práticas terapêuticas. Por exemplo, Kazdin (1978) ao discutir questões, recentes à época, a respeito da técnica de dessensibilização sistemática, um dos componentes centrais da então incipiente Terapia Comportamental, comenta: "Devido à eficácia da dessensibilização ter sido bem estabelecido, uma questão maior não é o poder da técnica, mas os mecanismos através dos quais ela opera." (p. 214, tradução nossa). Sob controle das mesmas preocupações, Baer, Wolf e Risley (1968), ao delinear as setes dimensões de um estudo aplicado, postulam a necessidade de descrever precisamente os procedimentos (dimensão tecnológica), eviden- ciar as variáveis responsáveis pela mudança comportamental (dimensão analítica) e relacioná-las aos conceitos da área de maneira conceitualmente consistente (dimensão conceitual). O desafio em responder ao "por quê" da possível efetividade de tratamentos clínicos (Barnes-Holmes et al., 2016) permanece uma questão contemporânea pois, aparentemente, não foi satisfatoriamente respondida.

O afastamento dos princípios filosóficos, conceituais e metodológicos da Análise do Comportamento, parecem ser elementos que contribuíram para que a ACT ainda enfrente dilemas persistentes: seus vários procedimentos e técnicas, supostamente eficazes na produção de mudanças clínicas, ainda que interpretados por princípios comportamentais e influenciados pela RFT, teriam mecanismos através dos quais operam ainda obscuros (Almeida, Guedes \& Santos, 2020; BarnesHolmes et al., 2016).

A questão contemporânea de identificar os processos comportamentais básicos, envolvidos nos procedimentos da ACT, principalmente aqueles descritos pela RFT, portanto, alinham-se à preocupação behaviorista radical em esclarecer conceitualmente os processos básicos envolvidos em intervenções efetivas, e submetê-los à investigação experimental. Para os propósitos do presente artigo, duas das principais tentativas de investigação dos princípios básicos envolvidos na desfusão cognitiva serão discutidas: (i) a análise interpretativa e (ii) a análise experimental.

\section{Investigação Interpretativa}

Historicamente, a desfusão cognitiva originou-se quando a técnica cognitiva do distanciamento cognitivo foi apropriada por terapeutas comportamentais, e então reinterpretada conforme os princípios analítico-comportamentais sob o título de distanciamento compreensivo (Blackledge \& Drake, 2013; Zettle, 2011). Conforme Blackledge e Drake (2013), esta interpretação inicial propunha que determinadas contingências fortaleciam a formulação e seguimento rígido de regras, e para enfraquecer o controle verbal, a estratégia do distanciamento compreensivo consistia em estabelecer contingências que aumentavam a probabilidade de 
ocorrência de comportamentos inconsistentes com regras. Posteriormente, com a produção de estudos de eficácia (Zettle \& Hayes, 1986), bem como de pesquisas básicas da RFT, a técnica foi novamente reinterpretada nos termos do responder relacional arbitrariamente aplicável.

Considerando que o RRAA está sujeito ao controle por contextos funcionais, de acordo com Blackledge (2003), as inúmeras variáveis controladoras da transformação de função entre eventos relacionados passam a constituir o denominado contexto de literalidade. Nas palavras do autor, "O contexto de literalidade se refere ao suporte contínuo, no formato do reforçamento diferencial fornecido pela comunidade sócio verbal, para a transformação de funções de estímulos que ocorrem durante o responder relacional derivado arbitrariamente aplicável." (p. 431, tradução nossa). Nesse sentido, o contexto de literalidade, responsável pelo controle da transformação de função, envolveria as mais diversas contingências sociais sob as quais o responder à funções derivadas é consistentemente reforçado. Entre as variáveis que compõe este contexto, Blackledge (2007) aponta características linguísticas como estruturas gramaticais e sintáticas, o uso de determinadas palavras para especificar e relacionar estímulos, e a velocidade e estilo de fala típico de uma cultura.

Em virtude da pervasividade deste contexto, a transformação de função de estímulos ocorre continuamente e em alta frequência, de tal forma que comportamentos podem passar a ocorrer sob rígido controle de funções verbalmente adquiridas pelos mais diversos eventos ambientais. $\mathrm{Na}$ terminologia da ACT, este controle rígido do comportamento por funções derivadas é denominado fusão cognitiva (Blackledge, 2007; Blackledge \& BarnesHolmes, 2009; Hayes et al., 2012a).

Partindo de tais premissas, muitas reconceitualizações da desfusão cognitiva foram propostas em conformidade com o referencial da RFT. Entre as tantas definições, destaca-se a de Blackledge e Barnes-Holmes (2009), segundo a qual a desfusão cognitiva é entendida como

um processo no qual as transformações de estímulos verbais bem estabelecidos são interrompidas através do deslocamento de condi- ções que controlam o responder relacional em geral[...] em momento em que condições como estas são deslocadas, acredita-se que ocorra a interrupção das transformações de funções verbais estabelecidas por meio da fusão. (pp. 49-50, tradução nossa).

Similarmente, Blackledge (2007) afirma que fusão cognitiva se refere aos contextos nos quais transformações verbais de funções de estímulos estão prontamente ocorrendo, enquanto desfusão $\operatorname{cog}$ nitiva se refere aos contextos nos quais estas transformações verbais são, ao menos temporariamente, interrompidas." (p.557, tradução nossa). Dito de outra forma, a desfusão seria uma forma de "violar os parâmetros de uso da linguagem" (Blackledge, 2007, p. 562, tradução nossa), de maneira que as palavras percam seu controle sobre o comportamento subsequente. Uma vez que a transformação de função é interrompida, a desfusão cognitiva permitiria que funções diretas de estímulos exerçam controle sobre o responder. Salienta-se que a proposta de Blackledge (2007) é uma entre muitas interpretações baseadas nos conceitos básicos da RFT a respeito da desfusão cognitiva. Tal será a definição sustentada ao longo do artigo.

Aqui, é importante deixar clara a distinção entre procedimento, processo e resultado. $\mathrm{O}$ termo procedimento refere-se à manipulações ambientais - em termos experimentais, à mudanças na variável independente; o processo, diz respeito à mudanças no responder produzidas pelo procedimento; por fim, resultado diz respeito à estabilização do responder decorrente do processo (Assaz et al., 2018; Barnes-Holmes et al., 2016). Nesse sentido, conforme ambas definições, compreende-se que, tecnicamente, a desfusão cognitiva envolveria o (i) procedimento de manipulação (deslocamento, interrupção ou enfraquecimento) de condições contextuais responsáveis pela transformação de função de estímulos, de tal maneira que seguir-se-ia (ii) o processo de interrupção ou atenuação da transformação de função entre estímulos relacionados, gerando, como (iii) resultado a diminuição de comportamentos controlados por funções derivadas. Portanto, o termo médio "desfusão cognitiva" estaria se referindo à relações funcionais entre organismo e ambiente teorizadas pela RFT. 
Esta interpretação sustenta-se em estudos experimentais que demonstraram o controle contextual sobre a transformação de função de estímulos (Barnes, Browne, Smeets, \& Roche, 1995; Roche, Barnes-Holmes, Smeets, Barnes-Holmes, \& McGeady, 2000; Perez, Fidalgo, Kovac, \& Nico, 2015). Todos os estudos mostraram claramente que funções de estímulos específicas - e.g. reforçadores, eliciativas, evocativas - podem ser transformadas de acordo com a manipulação de variáveis contextuais específicas. Os dados atualmente disponíveis validam a possibilidade de alterar a transformação de função através de variáveis contextuais (Dougher, Perkins, Greenway, Koons \& Chiasson, 2002; Gomes et al., 2019), mas não especificamente a interrupção deste processo via manipulação das mesmas condições contextuais (de Rose, 1993). Há, portanto, um descompasso entre a interpretação do termo médio desfusão e os dados experimentais sob os quais a interpretação foi construída.

Adicionalmente, ainda que as definições discutidas acima proponham a interrupção ou atenuação de transformação de função como o processo envolvido na desfusão cognitiva, é relevante apontar que ainda não há clareza a respeito dos processos comportamentais através dos quais as várias estratégias clínicas de desfusão atuam. Em uma análise conceitual de técnicas de desfusão cognitiva, Assaz et al. (2018) concluíram que a redução de respostas sob controle de funções derivadas, enquanto resultado comportamental, poderia ser produzido mediante diferentes procedimentos, e que cada qual opera através de diferentes processos comportamentais. Conforme a intepretação dos autores, exercícios de repetição ou manipulação de palavras envolveria o procedimento de exposição, produzindo mudanças comportamentais através dos processos de extinção respondente ou contra condicionamento; intervenções que visam romper a relação de causalidade entre pensamentos (eventos verbais) e ações, expondo o cliente a eventos privados e evocando respostas alternativas, corresponderia a um procedimento de reforçamento de respostas alternativas (DRA) e ao processo de reforçamento diferencial; por fim, exercícios que envolvem (i) recontextualizar pensamentos enquanto meras narrativas e metáforas, e (ii) estabelecer relações de perspectiva espacialmente distantes entre pensa- mentos e observador, envolveriam o procedimento de alteração dos contextos nos quais o responder à relações ocorrem. Curiosamente, apenas o último conjunto de técnicas corresponderia ao procedimento que define a desfusão cognitiva conforme teorização de Blackledge e Barnes-Holmes (2009) em termos da RFT.

Considerando tal percurso, pode-se afirmar que a relação entre o termo médio desfusão cogniti$v a$, e os processos comportamentais descritos pela RFT foi, tanto em sua origem quanto em sua concepção atual, estabelecida via análise interpretativa. Para a análise do comportamento, a interpretação é uma forma legítima de produção de conhecimento, uma vez que envolve à utilização de conceitos extraídos da pesquisa empírica para a explicação de fenômenos cuja complexidade impede a investigação experimental (Donahoe, 1993). Ainda assim, uma interpretação para a desfusão cognitiva nos termos da RFT não é suficiente para preencher a lacuna existente entre termos médios, os procedimentos por eles nomeados e a pesquisa básica: definições de conceitos construídas dessa forma devem ser consideradas com cautela em razão do risco de constituírem extrapolações dos dados experimentais - tal parece ser o caso da definição dada por Blackledge e Barnes-Holmes (2009) e os estudos sobre os quais se baseia.

As conclusões de Assaz et al. (2018) deixam claro o nível de imprecisão conceitual e discrepância entre teoria e prática resultantes da estratégia reticulada de produção de conhecimento defendida por Hayes et al. (2012b). Conforme a proposta da CBS, investigação aplicada, conceitual e experimental, bem como a reticulação entre estes domínios, são igualmente relevantes, e não se deve pressupor a primazia de um sobre o outro. Alinhado a esta proposta, as contribuições de Assaz et al. (2018) favorecem o diálogo entre dimensão aplicada e conceitual; entretanto, a reticulação destes domínios com a investigação experimental ainda carece de esforços adicionais.

Ainda no que tange à interpretação, BarnesHolmes et al. (2016) denunciam que é cada vez maior a prática de utilizar princípios bem estabelecidos da RFT para "traduzir" termos médios, prática esta considerada insuficiente para uma adequada análise do responder relacional. Apesar destas in- 
terpretações aparentarem coerência com a terminologia básica, argumentam os autores, não conduzem, necessariamente, à análises experimentais do responder relacional. Por exemplo, até o presente momento, não existe nenhuma pesquisa básica análoga que reproduza e permita observar diretamente o responder relacional descrito na definição de desfusão cognitiva discutida acima. Conforme será discutido à diante, existem pesquisas dedicadas à verificação experimental de estratégias de desfusão sem, entretanto, possibilitar a reprodução de relações sistemáticas entre responder relacional e manipulações contextuais alinhadas a conceitualização da estratégia. As definições de Blackledge e Barnes-Holmes (2009), embora coerente com os princípios da RFT, parece não ter despertado a curiosidade de pesquisadores básicos em delinear experimentos capazes de validar empiricamente o conceito. É nesse sentido que Barnes-Holmes et al. (2016) defendem o posicionamento de que a evolução de uma ciência não pode se basear unicamente em interpretações dos fenômenos clínicos descritos por termos médios através de outros termos funcionalmente precisos: "Interpretações da RFT que permanecem nada além de interpretações, têm pouco mais a oferecer do que confiar exclusivamente em termos médios RFT.' (p. 377, tradução nossa). Em resumo, ainda que a interpretação possibilite uma definição do conceito utilizado pelo cientista de maneira coerente com os princípios básicos, a distância entre dados experimentais e os termos médios permanece.

\section{Investigação Experimental}

Voltar a atenção à pesquisa básica afim de esclarecer empírica e conceitualmente a qual fenômeno os termos médios se referem não se limita a uma preocupação puramente teórica. Confusão na teoria, afirma Skinner (1953/2003), implica em confusão na prática, e as confusões conceituais por parte dos praticantes prejudicam ainda mais o desenvolvimento de novas aplicações tecnológicas. Por exemplo, o praticante pode utilizar-se de um procedimento efetivo em produzir mudança comportamental; entretanto, a generalidade dos resultados é comprometida quando o terapeuta não é capaz de relacionar as variáveis independentes manipuladas aos conceitos básicos (Guilhardi, 2002; Michael, 1980). Problemas práticos decorrentes de imprecisões conceituais já foram denunciadas por autores contemporâneos. Barnes-Holmes et al. (2016) apontam para uma frequente confusão por parte de praticantes de ACT em relação à diferenciação da desfusão enquanto procedimento, processo e resultado. Um exemplo de circularidade decorrente dessa confusão seria o caso em que um clínico identifica que o cliente se encontra "fusionado" com pensamentos negativos, e, portanto, utiliza técnicas de desfusão (procedimentos) para ativar o processo de desfusão e então produzir um repertório desfusionado (resultado). Uma vez que um mesmo termo é utilizado para se referir a diferentes fenômenos, a descrição do que é manipulado e o que é modificado perde a coerência conceitual e assume a circularidade. Definir precisamente, em conformidade com o jargão teórico e experimental adotado, à quais processos, procedimento ou resultados um termo médio se refere é de relevância prática - e a pesquisa básica é um caminho para cumprir esta tarefa.

Levin e Villatte (2016) apontam para duas direções tomadas pelos estudos de laboratório, conforme orientados pela proposta da CBS, voltados à aproximação entre termos médios e processos comportamentais básicos.

Em primeiro lugar, encontram-se as pesquisas dedicadas à verificar, em contexto experimental, se estratégias de intervenção que compõe o modelo da ACT apresentam efeitos consistentes com o modelo teórico subjacente. Estudos dessa natureza podem ser entendidos como uma aproximação entre as dimensões aplicada e experimental de produção de conhecimento. De maneira geral, o delineamento destas pesquisas envolve seleção e aplicação de componentes terapêuticos isolados ou em combinações específicas, e a verificação dos seus efeitos sobre (i) comportamentos clinicamente relevantes, quando mais próximas do continuum aplicado, ou sobre (ii) análogos clínicos ou medidas de processos, quando mais próximas do continuum básico - estes, têm sido denominados, na literatura, de análogos experimentais.

A literatura conta com uma grande variedade de produções de pesquisas análogas, vinculadas 
à investigação da conexão entre RFT e estratégias da ACT, como a desfusão cognitiva (Donati et al., 2019; Ferroni-Bast, Fitzpatrick, Stewart \& Goyos, 2019; Gil-Luciano, Ruiz, Valdivia-Salas \& SuárezFalcón, 2017; Kishita, Muto, Ohtsuki \& BarnesHolmes, 2014; López-López \& Luciano, 2007; Luciano et al., 2014; Masuda, Hayes, Sackett \& Twohig, 2004), aceitação (Keogh, Bond, Haner \& Tilson, 2005; Mcmullen, Barnes-Holmes, BarnesHolmes, Stewart \& Cochrane, 2007) e promoção de self contextual (Foody, Barnes-Holmes, BarnesHolmes, Rai \& Luciano, 2013; Foody, BarnesHolmes, Barnes-Holmes, Rai \& Luciano, 2015). Apesar da variedade de estudos, para os propósitos do presente trabalho, será dada uma ênfase à investigação da desfusão cognitiva.

Como apontou Assaz (2019), estes estudos análogos apresentam um elevado grau de validade interna em virtude do controle sobre as variáveis independentes, uma vez que permitem que o experimentador identifique precisamente se o componente apresentado aos participantes é ou não responsável pela ocorrência das variações nas variáveis dependentes. Ainda que sejam baseadas e até disporem de interpretações coerentes com a RFT, os delineamentos experimentais empregados neste tipo de pesquisa, apesar do rigor experimental, não permitem uma análise dos processos básicos envolvidos no fenômeno observado, uma vez que não permitem isolar precisamente as variáveis manipuladas durante os estudos. Sua contribuição, portanto, está mais vinculada à produção de dados acerca da efetividade de técnicas compreendidas pelos termos médios do que com uma conceitualização consistente e demonstração dos processos básicos dos fenômenos referidos por estes termos. Aqui, a seleção de variáveis dependentes e independentes são os pontos críticos que inviabilizam o controle do fenômeno em um nível básico.

Em relação às variáveis dependentes, alguns estudos buscam avaliar o efeito das estratégias de desfusão em escores obtidos em inventários de autorrelatos, os quais supostamente medem processos comportamentais. Da mesma forma, pesquisas mediacionais utilizam tanto questionários que supostamente avaliam o processo de desfusão quanto medidas que supostamente avaliam os resultados comportamentais (e.g. inventários de credibilida- de de pensamentos) do procedimento; através de tratamentos estatísticos, considera-se que as mudanças em uma medida de processo são as responsáveis pelas mudanças nas medidas de resultado. A utilização dessas formas de mensuração também é insuficiente, uma vez que medidas psicométricas de processos não descrevem precisamente processos funcionais, não permitem uma observação direta do responder relacional, e as conclusões permanecem correlacionais e interpretativas (Dymond et al, 2013; Barnes-Holmes et al., 2016; Levin \& Villatte, 2016); nas palavras de Barnes-Holmes et al. (2016), estes inventários não são capazes de mensurar mudanças no responder relacional, mas sim "o comportamento de preencher questionários ou completar um diário (p. 370, tradução nossa)". Novamente, estas preocupações já eram apontadas por Baer, Wolf e Risley (1968), quando, entre os critérios para uma pesquisa aplicada, enfatiza-se a necessidade de mensuração precisa da variável dependente (dimensão comportamental).

É importante salientar que um maior grau de controle experimental é atingido nas pesquisas que verificam o efeito de estratégias de desfusão tendo medidas de comportamentos diretamente observáveis pelo pesquisador nos valores da $\mathrm{VD}$, como por exemplo, o comportamento de continuar em contato com estimulação aversivas ou desempenhar tarefas nestas condições (Gil-Luciano et al., 2017; Keogh et al., 2005; López-López \& Luciano, 2017; McMullen et al., 2007), desempenho em tarefas que mensuram atenção e coordenação motora (LópezLópez \& Luciano, 2017), latência de respostas relacionais mensurada através do IRAP (Kishita et al. 2014; Ferroni-Bast et al., 2019) e o responder de esquiva derivada a um estimulo participante de uma classe de equivalência (Donati et al., 2019; Luciano et al., 2014). O estudo que parece ter obtido maior controle experimental em relação ao estabelecimento e emergência do responder relacional clinicamente relevante foi o análogo de desfusão cognitiva de Donati et al. (2019). Os autores verificam o efeito de um procedimento análogo desfusão e reestruturação cognitiva sobre uma resposta de esquiva sob controle derivado experimentalmente estabelecida. De maneira geral, foi construída uma classe de equivalência de três membros (A1-B1-C1); em seguida, através de condicionamento respondente $\mathrm{e}$ 
operante, foi estabelecida função aversiva e evocativa para uma resposta de esquiva ao estímulo B1, seguido por um teste de transferência de função para C1. Aqui, as respostas de esquiva derivada ao estímulo $\mathrm{C} 1$ foram consideradas análogas à esquiva experiencial de um evento verbal, devido à fusão cognitiva. $\mathrm{Na}$ fase experimental, um dos grupos de participantes recebeu um protocolo adaptado do exercício de desfusão de repetição de palavras. Os participantes foram instruídos a repetir a palavra "milk" e, em seguida, fazer o mesmo exercício com C1 (no caso, uma palavra sem sentido) por sete minutos e meio. Por fim, verificou-se os efeitos do procedimento sobre a ocorrência esquiva derivada em uma segunda exposição ao estímulo C1. O estudo de Donati et al. (2019) destaca-se pelas escolhas metodológicas: pode-se dizer que os procedimentos empregados consistiram em um esforço em "construir" totalmente a variável dependente.

Ainda assim, a utilização de medidas ou mesmo padrões de comportamentos construídos em laboratório não é suficiente para permitir, aos análogos experimentais, a verificação de processos comportamentais básicos quando as variáveis independentes não apresentam o mesmo cuidado de controle experimental em relação à sua construção. Por exemplo, Gil-Luciano et al. (2017) buscaram verificar o efeito de um protocolo de desfusão com molduras dêiticas ou hierárquicas sobre a tolerância na pressão ao gelo e tempo de exposição a um filme aversivo. O protocolo apresentado ao grupo experimental envolveu uma interação de aproximadamente 20 a 30 minutos, no qual o experimentador passou instruções ao participante, como imaginar-se maior que a dor, encontrar um lugar para dor, etc. Aqui, o controle experimental foi prejudicado pois as funções comportamentais em vigor em cada parcela das frases apresentadas não foram construídas em laboratório; os experimentadores inferiram, a partir do uso cotidiano, que os termos utilizados (imagine-se maior que) funcionariam como dicas contextuais para o responder dêitico e hierárquico. As mesmas questões também estão presentes no estudo de Donati et al. (2019): apesar do elevado grau de controle experimental sobre a variável dependente, os autores utilizaram, como variável independente, um procedimento desfusão derivado de um protocolo clínico. Em todos os casos, trata-se de variáveis independentes cujo grau de complexidade vai além daquele que se busca na pesquisa básica.

A ausência de controle histórico e experimental sobre a variável independente não permite identificar precisamente quais funções comportamentais a variável assume, qual dos seus aspectos foram responsáveis pelas mudanças comportamentais que se seguem, e quais são os processos básicos em curso desencadeados pelo procedimento. Com isso, o uso dessas estratégias clínicas enquanto variável independente em estudos análogos pode implicar não só em falhas na identificação dos processos comportamentais, como também em imprecisões quanto à quais processos se devem os resultados comportamentais obtidos.

Uma outra direção tomada pelas pesquisas de laboratório são investigações experimentais direcionadas à aproximação entre fenômenos clínicos e princípios comportamentais básicos (Levin \& Villatte, 2016). Estudos deste tipo partem do pressuposto de que o fenômeno básico experimentalmente estudado está vinculado à fenômenos complexos observados em situações práticas. Em relação às escolhas metodológicas, há um grande cuidado em garantir o completo controle experimental tanto sobre as variáveis manipuladas, quanto pela mensuração precisa do comportamento de interesse, condições estas que não podem ser atingidas pelas pesquisas translacionais, de processo-resultado e estudos de componente (Levin \& Villatte, 2016). De acordo com Dymond et al. (2013), tais estudos envolvem procedimentos para o treino e derivação de redes relacionais, bem como para a transformação de funções de estímulos a fim a estabelecer ou modificar um responder análogo ao tratamento de comportamentos clinicamente relevantes. Como aponta Dymond et al. (2013), ainda há uma quantia limitada de pesquisas dessa natureza, cujo foco principal consiste em análogos de medo e esquiva.

Um exemplo é o trabalho de Roche, Kanter, Brown, Simon e Fogarty (2008), na qual os autores buscaram comparar o efeito do procedimento de extinção de esquiva de funções adquiridas diretamente ou por derivação. Para estabelecer o comportamento de esquiva análogo, inicialmente, os participantes passaram por um treino no qual foi estabelecida uma rede relacional entre cinco estí- 
mulos (B1 igual C1; B2 igual C2; B1 oposto C2 e B1 oposto C1). Em seguida, foi estabelecida a função evocativa de esquiva para B1, e verificou-se a derivação da função para o estímulo $\mathrm{C} 1$. Os participantes foram divididos em dois grupos: o primeiro, passou por um procedimento de extinção diante B1 (extinção direta); o segundo grupo foi exposto à extinção diante $\mathrm{C} 1$ (extinção derivada). Em seguida, os participantes foram expostos novamente aos estímulos B1 e C1. Como resultado, a condição extinção derivada produziu menor porcentagem de esquivas para $\mathrm{B} 1$ e $\mathrm{C} 1$ do que a condição direta.

O que se destaca no estudo de Roche et al. (2008) diz respeito à seleção e controle experimental sobre as variáveis manipuladas. Os pesquisadores garantiram a reprodução análoga em laboratório tanto de uma intervenção - exposição - quanto de um comportamento clinicamente relevante - esquiva a funções aversivas diretas ou derivadas, característica do quadro de ansiedade. Ademais, apesar da pesquisa não se propor de antemão ao estudo da desfusão, a partir dos dados obtidos, levantou-se a hipótese de que os exercícios de desfusão cognitiva constituídos por repetição de palavras (e.g. "milk, milk, milk") envolveriam os processos investigados neste estudo. Isto é, repetição de palavras - pensamentos desagradáveis - corresponderia a um procedimento de extinção das funções aversivas derivadas adquiridos por este estímulo; com isso, a extinção é transferida para demais estímulos relacionados à palavra, as quais deixam de exercer o controle derivado.

Apesar de desempenhar um papel relevante tanto na prática terapêutica quanto na própria história da ACT, a desfusão cognitiva tem ganhado, no campo da pesquisa de laboratório, muito mais atenção em pesquisas de eficácia de componentes do que em tentativas de esclarecimento dos processos comportamentais básicos envolvidos nesta intervenção. $\mathrm{O}$ excesso de estudos do primeiro tipo e a ausência do segundo implicam na sustentação de uma técnica "efetiva", mas cujos mecanismos responsáveis pela efetividade permanecem desconhecidos. Ainda que disponha de interpretações da RFT para a desfusão cognitiva, a literatura ainda carece de estudos empíricos planejados com delineamentos que permitam esclarecer, em termos de princípios básicos, as relações funcionais relacionadas ao termo.

\section{Direções para a Pesquisa Experimental}

Se, conforme postula Hayes et al. (2012b), uma completa abordagem científica exige "um esforço constante para que os termos médios sejam gradualmente ancorados em abordagens mais técnicas" (p.7), então uma direção promissora para investigações futuras é o retorno à pesquisa básica, à forma de produção de conhecimento originalmente proposto pela Análise do Comportamento. Com efeito, existe um grande volume de pesquisas experimentais rigorosas demonstrando os princípios mais básicos da RFT - implicação mútua e combinatória, transformação de funções -; o que é criticado é a ausência de estudos de laboratório que demonstrem relações confiáveis entre variáveis independente e dependentes na investigação dos processos envolvidos em intervenções teoricamente baseadas na RFT.

Dymond et al. (2013) defendem um "padrão ouro" para a pesquisa experimental acerca do responder relacional envolvido em intervenções clínicas ou psicopatologias: a condução de estudos cuidadosamente planejados, de tal forma que o delineamento experimental permita o "completo controle experimental sobre o próprio processo relacional, durante a emergência e tratamento análogo de comportamentos clinicamente relevantes" (p. 201, tradução nossa). Os autores propõem que pesquisas deste tipo utilize procedimentos para o treino e derivação de redes relacionais e transformações de função, tanto para o estabelecimento quanto diminuição de comportamentos análogos aos processos psicopatológicos.

No que tange às direções para a pesquisa experimental, o que o presente artigo propõe é voltar o foco a investigações experimentais dos eventos referidos pelos termos médios e demais definições interpretativas por meio de análogos de fenômenos e intervenções clínicas, delineados de tal forma que seja garantido o completo e rigoroso controle experimental sobre as variáveis dependentes e independentes. Como já foi discutido acima, a literatura já conta com um extenso engajamento na condução de análogos experimentais dedicados à investigação de procedimentos clínicos sobre análogos de com- 
portamentos problemáticos, entretanto, os delineamentos frequentemente empregados implicam, em virtude da natureza das variáveis independentes estudadas, nas limitações já apontadas no que tange à contribuição com o esclarecimento de procedimentos e processos comportamentais básicos.

Quando o objetivo do estudo é o esclarecimento de processos básicos subjacentes à fenômenos complexos, o total controle sobre as variáveis é imprescindível. Nesse sentido, o cuidado que aqui se propõe diz respeito a construir em contexto de laboratório tanto as variáveis independentes quanto dependentes análogas às intervenções $e$ comportamentos clinicamente relevantes.

Estudos dessa natureza permitiriam (i) testar empiricamente a precisão de interpretações de intervenções, (ii) construir e isolar, em contexto experimental, as variáveis independentes manipuladas na desfusão cognitiva (bem como outros componentes da ACT), a (iii) observar o responder relacional "in vivo" envolvido, e a (iv) verificar se os seus efeitos sobre o comportamento correspondem àqueles teoricamente previstos. Salientase que a presente proposta se trata de retornar às direções metodológicas originais da Análise do Comportamento para a investigação de processos comportamentais básicos e derivação de tecnologias, quais sejam, pesquisa básica guiada pela descrição, mensuração e controle rigoroso das variáveis independentes e dependentes (Johnston \& Pennypacker, 2009; Sidman, 1960).

Antes de propor os cuidados ao qual o experimentador deve se atentar, vale a pena ressaltar as críticas tecidas a detalhes dos delineamentos geralmente utilizados em estudos de análogos, os quais devem ser evitados.

Barnes-Holmes et al. (2016) aponta um conjunto de erros cometidos por pesquisadores na construção de delineamentos para análogos experimentais de desfusão cognitiva. O primeiro erro consiste em (i) selecionar como variável independente uma estratégia terapêutica completa, convencionalmente considerada um procedimento de desfusão direcionado a um processo de desfusão. Como consequência do primeiro erro, o (ii) segundo erro diz respeito à assumir que existe um processo funcional de desfusão. O (iii) terceiro erro está em considerar que a aplicação daquele procedimento irá necessariamente fornecer uma evidência direta do processo funcional de desfusão. Com a condução do experimento, o (iv) quarto erro consiste em considerar que a observação de uma relação entre o procedimento e o resultado obtido corresponde ao processo de desfusão, e não algum outro processo comportamental. Por fim, o (v) quinto erro consiste em considerar que a produção de resultados semelhantes por procedimentos diferentes corrobora a hipótese de que existe um processo de desfusão (Barnes-Holmes et al., 2016).

Adicionalmente, considerando que a investigação experimental deve se voltar à identificação de processos comportamentais básicos (Andery, 2010; Johnston \& Pennypacker, 2009; Matos, 1990; Sampaio et al., 2008; Sidman, 1960; Velasco, GarciaMijares, \& Tomanari, 2010), é importante salientar que não se deve voltar à busca de um "processo de desfusão", mas sim a descrições de relações funcionais entre as variáveis dependentes e independentes observadas quando se reproduz experimentalmente o fenômeno de interesse. Se, conforme Skinner (1966), “o processo comportamental estudado em uma análise experimental usualmente consiste em mudanças na probabilidade (ou taxa de resposta) como uma função de variáveis manipuladas" (p. 216, tradução nossa), então no estudo das características do responder relacional envolvido na desfusão cognitiva, o processo comportamental a ser investigado consiste em mudanças na probabilidade de respostas sob controle derivado e alterações em demais propriedades do RRAA experimentalmente estudadas, como latência (Barnes-Holmes, BarnesHolmes, Luciano, \& McEnteggart, 2017) e grau de transformação de função (de Almeida, Bortoloti, Ferreira, Schelini , \& de Rose, 2014; Santos, Perez, de Almeida \& de Rose, 2017), como uma função da manipulação de um contexto funcional.

Outro detalhe relevante diz respeito ao delineamento experimental e estratégia de análise de dados. Conforme Johnston \& Pennypacker (2009), é sempre a pergunta experimental que deve orientar a escolha do cientista acerca da natureza do delineamento e análise de dados empregado. Em relação às pesquisas análogas conduzidas aos moldes da CBS, a grande maioria recorre ao delineamento de grupo e ao uso de estatística inferencial. Uma vez que alinhadas à preocupação em garantir 
o controle experimental, estas escolhas metodológicas são justificadas e contribuem com o objetivo do estudo. Por exemplo, em análogos de desfusão com delineamentos entre sujeitos, testes estatísticos são imprescindíveis para que se verifique se, após as manipulações, os valores assumidos pelas variáveis dependentes foram estatisticamente diferentes entre os grupos controle e experimental.

Por outro lado, é importante apontar algumas das limitações destas metodologias quando o interesse do pesquisador está na observação de particularidades de processos comportamentais básicos. Nestes casos, defende-se uma preferência pelo delineamento de sujeito único. Relações comportamentais são fenômenos que ocorrem em organismos individuais, e nenhum organismo responde exatamente da mesma forma às condições a que está exposto (Andery, 2010; Johnston \& Pennypacker, 2009). A observação dessas relações, portanto, pode ser facilitada através do delineamento de sujeito único, uma vez que permite produzir dados acerca de variações do responder de um mesmo indivíduo conforme exposto à diferentes valores da variável independente.

Em relação ao tratamento dos dados, a estatística inferencial pode informar ao experimentador se a variável independente afeta a variável dependente, ou em que medida ambas estão relacionadas; entretanto, o interesse da pesquisa experimental vai além: diz respeito a observar as mudanças no fluxo comportamental e a avaliar mudanças e estabilidades ao longo do tempo (Andery, 2010; Hopkins, Cole, \& Mason, 1998). As variáveis manipuladas sempre afetam o comportamento de indivíduos, e limitar os dados ao tratamento estatístico não permite que o experimentador identifique de que maneira o responder foi modificado ao longo de diferentes condições. Em contrapartida, o tratamento gráfico de dados obtidos via delineamento de sujeito único, no qual o mesmo organismo é exposto à condição controle e experimental, permite que o experimentador observe cuidadosamente as alterações na regularidade e nas dimensões comportamentais que seguem às manipulações (Andery, 2010; Johnston \& Pennypacker, 2009; Sampaio et al., 2008; Velasco et al., 2010). Não se propõe, contudo, um abandono completo da estatística, mas sim a sua utilização enquanto recurso comple- mentar à análise individual e gráfica; como afirma Andery (2010), "o problema existe quando e se a quantificação tornar o grande objetivo da pesquisa (p. 332)". O objetivo da pesquisa experimental sempre é a descrição de relações funcionais entre variáveis independente e dependentes, e os dados obtidos através da análise estatística são complementares, e não substitutos aos dados gráficos.

No que diz respeito à seleção e descrição das variáveis dependentes e independentes, a pesquisa de Roche et al. (2008) citada acima é um bom exemplo do controle rigoroso proposto para o estudo do responder relacional. Até a presente data, nenhum análogo direcionado à investigação dos processos básicos envolvidos na desfusão cognitiva, conhecido pelos autores, seguiu o mesmo nível de controle experimental em relação às variáveis independente e dependente atingido por Roche et al. (2008) no estudo dos processos básicos envolvidos na exposição.

A pesquisa de desfusão que mais se aproximou do padrão ouro proposta por Dymond et al. (2013) foi, como já discutido, o experimento de Donati (2019). A variável dependente, assim como na pesquisa de Roche et al. (2008), foi uma resposta de esquiva sob controle de funções derivadas adquiridas por um estímulo por participar de uma classe de equivalência. Entretanto, os autores falharam em construir experimentalmente uma variável independente análoga, recorrendo à um protocolo clínico (exercício de repetição de palavras).

Um análogo experimental padrão ouro exigiria construir em laboratório tantos as variáveis dependentes quanto independentes análogas ao fenômeno de interesse, e investigar suas relações funcionais em delineamentos de sujeito único. Um estudo coerente com a presente proposta envolveria não só a reprodução de um análogo do comportamento de esquiva derivada, mas também, ao invés da aplicação de uma estratégia clínica, um análogo experimental das variáveis manipuladas nos procedimentos de desfusão. Se, conforme a definição de Blackledge e Barnes-Holmes (2009), a desfusão se refere ao procedimento de manipulação de uma condição contextual controladora da transformação de função, é esta variável que deve ser construída em um estudo análogo. O experimento como um todo consistiria em inserir e retirar esta condi- 
ção contextual, e verificar as mudanças - processos e resultados - que se seguem em uma resposta de esquiva derivada. Atualmente, os presentes autores têm conduzido estudos nesta direção.

\section{Considerações finais}

O modelo reticulado de investigação científica proposto pela CBS diverge da forma de produção de conhecimento originalmente adotado pela Análise do Comportamento. O objetivo principal do presente artigo consistiu em discutir as fragilidades decorrentes da forma como o modelo reticulado foi colocado em prática no que tange à investigação dos princípios comportamentais básicos estudados pela RFT, no desenvolvimento tecnológico da ACT, e das interações teórico-experimentais entre ambos os domínios. É importante enfatizar que estas fragilidades não são características intrínsecas à proposta conceitual de um modelo reticulado, mas sim derivações da forma como foi colocado em prática: em tese, o modelo propõe uma interação constante entre as dimensões teórica, básica e aplicada - mas esta interação não tem sido efetivamente praticada.

As principais fragilidades destacadas foram o distanciamento entre os procedimentos da ACT e a base teórico-experimental da RFT, e a consequente falta de precisão dos termos médios. No que diz respeito à desfusão cognitiva, a falta de precisão é um resultado do emprego da estratégia reticulada desde o princípio da sua formulação. Cronologicamente, em sua origem, tratava-se de um procedimento supostamente efetivo - o distanciamento cognitivo -, mas alheio aos pressupostos behavioristas radicais, uma vez que originado enquanto parte de uma terapia cognitiva; o procedimento foi então ampliado e reinterpretado de acordo com princípios analítico-comportamentais skinnerianos; posteriormente à produção de evidências empíricas da RFT, a desfusão foi novamente reinterpretada nos termos do responder relacional arbitrariamente aplicável. Como resultado, as problemáticas decorrentes das imprecisões da desfusão cognitiva envolvem a referência à procedimentos diversos, confusão a respeito dos mecanismos através dos quais atua, circularidade ao ser utilizada para se referir à procedimento, processo e resultado, definições imprecisas e extra- poladas de pesquisas básicas, e pouca efetividade em orientar pesquisas experimentais básicas (Assaz et al., 2018; Barnes-Holmes et al., 2016; Dymond et al., 2013).

Partindo de tais argumentos, propõe-se que uma direção, no campo da pesquisa básica, para que termos médios atinjam maior grau de precisão é a investigação experimental dos princípios comportamentais envolvidos em questões aplicadas - procedimentos e fenômenos clínicos -, através da condução de pesquisas alinhadas aos cuidados metodológicos originais à Análise Experimental do Comportamento: delineamentos de sujeito único, ênfase no controle rigoroso e na mensuração a partir da observação direta das variáveis independentes e dependentes, descrições conceitualmente consistentes das relações funcionais sistemáticas observadas, e preferência pela análise gráfica ao invés do tratamento estatístico de dados (Johnston \& Pennypacker, 2009; Sidman, 1960) . Nas pesquisas de processos básicos da RFT, tal preocupação nunca esteve ausente; o que se enfatiza aqui é a importância de estendê-la também ao estudo dos processos básicos subjacentes a intervenções e fenômenos clínicos.

Os impactos das propostas e esforços da CBS na psicologia é inegável. Entretanto, ao declarar a sua independência, a CBS também deixou para trás aspectos característicos da Análise do Comportamento, pois este seria o custo para o desenvolvimento de uma "ciência comportamental mais adequada aos desafios da condição humana." (Hayes et al., 2012b, p.15, tradução nossa). Além de contribuir com a disseminação e adoção de tecnologias comportamentais por novos praticantes, é importante lembrar que esta cisão também contribuiu com a perda da precisão e rigor conceitual na produção de conhecimentos, teóricos e experimentais, acerca de fenômenos comportamentais.

É importante deixar claro que a posição assumida no presente artigo não é a de que a pesquisa de processos básico, independentemente de qualquer diálogo com as demais áreas, seja suficiente para resolução de demandas práticas - mas é considerada imprescindível. O ponto central foi enfatizar cuidados metodológicos e direções para o estudo experimental de fenômenos e intervenções clínicas, a fim de aumentar a precisão de termos médios e a 
confiabilidade das relações funcionais supostamente referidas por esses termos. Nesse sentido, sugere-se que um rumo mais promissor para garantir a reticulação almejada pela CBS envolve esforços direcionados à reaproximação do rigor experimental na pesquisa básica, conforme as orientações metodológicas da Análise Experimental do Comportamento, principalmente no que tange à interação entre dimensões básicas e aplicadas.

\section{Referências}

Almeida, P, Guedes, M. L, \& Santos, W. (2020). A prática clínica do analista do comportamento: possibilidades, desafios e uma necessária revisão de seus pressupostos. Em Almeida \& Guedes (org.), Análise do Comportamento no pós-graduação: pesquisas e reflexões do Programa de Psicologia Experimental da PUCSP (pp. 147-169). Ed CRV Curitiba.

Andery, M. A. P. A. (2010). Métodos de pesquisa em análise do comportamento. Psicologia USP, 21(2), 313-342. doi: 10.1590/S010365642010000200006

Assaz, D. A. (2019). Desfusão cognitiva na terapia de aceitação e compromisso (ACT): O processo de mudança clínica (Tese de Doutorado). Instituto de Psicologia, Universidade de São Paulo, São Paulo.

Assaz, D. A., Roche, B., Kanter, J. W., \& Oshiro, C. K. B. (2018). Cognitive Defusion in Acceptance and Commitment Therapy: What Are the Basic Processes of Change? Psychological Record, 68(4), 405-418. doi: 10.1007/s40732-0170254-Z

Baer, D. M., Wolf, M. M., \& Risley, T. R. (1968). Some current dimensions of applied behavior analysis. Journal of Applied Behavior Analysis, 1(1), 91-97. doi: 10.1901/jaba.1968.1-91

Barnes, D., Browne, M., Smeets, P. M., \& Roche, B. (1995). A transfer of functions and a conditional transfer of functions through equivalence relations in three to six year old children. Psychological Record, 45, 405-430. doi: 10.1007/ BF03395151

Barnes-Holmes, D., Barnes-Holmes, Y., Luciano, C., \& McEnteggart, C. (2017). From the IRAP and REC model to a multi-dimensional multi-level framework for analyzing the dynamics of arbitrarily applicable relational responding. Journal of Contextual Behavioral Science, 6(4), 434-445. doi: 10.1016/j.jcbs.2017.08.001

Barnes-Holmes, Y., Hussey, I., McEnteggart, C., Barnes-Holmes, D., \& Foody, M. (2016). Scientific ambition: The relationship between Relational Frame Theory and middle-level terms in Acceptance and Commitment Therapy. Em: R. D. Zettle, S. C. Hayes, D. Barnes-Holmes, \& A. Biglan (Eds), The Wiley handbook of contextual behavioral science (pp. 365-382), West Sussex, UK: Wiley-Blackwell.

Blackledge, J. T. (2003). An introduction to relational frame theory: Basics and applications. The Behavior Analyst Today, 3(4), 421-433. doi: 10.1037/h0099997

Blackledge, J. T. (2007). Disrupting verbal processes: Cognitive defusion in acceptance and commitment therapy and other mindfulness-based psychotherapies. Psychological Record, 57(4), 555-576. doi: 10.1007/BF03395595

Blackledge, J. T., \& Barnes-Holmes, D. (2009). Em: J. T. Blackledge, J. Ciarrochi, \& F. P. Deane (Eds.), Acceptance and Commitment Therapy - Contemporary Theory Research and Practice (pp. 41-59). Australian Academic Press.

Blackledge, J. T., \& Drake, C. E. (2013). Acceptance and Commitment Therapy: Empirical and Theoretical Consideations. Em S. Dymond \& B. Roche (Eds.), Advances in relational frame theory: Research and application (pp. 219-252). Oakland, CA: Context Press.

de Almeida, J. H., Bortoloti, R., dos Santos Ferreira, P. R., Schelini, P. W., \& de Rose, J. C. C. (2014). Análise da validade e precisão de instrumento de diferencial semântico. Psicologia: Reflexão e Crítica, 27(2), 272-281. doi: 10.1590/16787153.201427207

de Rose, J. C. (1993). Classes de estímulos: implicações para uma análise comportamental da cognição. Psicologia: Teoria e Pesquisa, 9(2), 283-303.

Donahoe, J. W. (1993). The unconventional wisdom of B. F. Skinner: the analysis-interpretation distinction. Journal of the Experimental Analysis of Behavior, 60(2), 453-456. doi: 10.1901/ jeab.1993.60-453 
Donati, M. R., Masuda, A., Schaefer, L. W., Cohen, L. L., Tone, E. B., \& Parrott, D. J. (2019). Laboratory analogue investigation of defusion and reappraisal strategies in the context of symbolically generalized avoidance. Journal of the Experimental Analysis of Behavior, 112(3), 225241. doi: 10.1002/jeab.550

dos Santos, G. A. R., Perez, W. F., de Almeida, J. H., \& de Rose, J. C. (2017). Transformação do significado de palavras sem sentido via relações arbitrárias de comparação com faces alegres. Perspectivas em Análise do Comportamento, 8(2), 269-285. doi: 10.18761/PAC.2017.028

Dougher, M., Perkins, D. R., Greenway, D., Koons, A., \& Chiasson, C. (2002). Contextual control of equivalence-based transformation of functions. Journal of the Experimental Analysis of Behavior, 78(1), 63-93. doi: 10.1901/jeab.2002.78-63

Dymond, S., Roche, B., \& Bennett, M. P. (2013). Relational frame theory and experimental psychopathology. Em: S. Dymond \& B. Roche (Eds.), Advances in relational frame theory: Research and application (pp. 199-217). Oakland, CA: Context Press.

Ferroni-Bast, D., Fitzpatrick, J., Stewart, I., \& Goyos, C. (2019). Using the Implicit Relational Assessment Procedure (IRAP) as a measure of reaction to perceived failure and the effects of a defusion intervention in this context. The Psychological Record, 69(4), 551-563. doi: 10.1007/s40732-019-00349-2

Foody, M., Barnes-Holmes, Y., Barnes-Holmes, D., \& Luciano, C. (2013). An empirical investigation of hierarchical versus distinction relations in a self-based ACT exercise. International Journal of Psychology and Psychological Therapy, 13(3), 373-388

Foody, M., Barnes-Holmes, Y., Barnes-Holmes, D., Rai, L., \& Luciano, C. (2015). An empirical investigation of the role of self, hierarchy, and distinction in a common act exercise. The Psychological Record, 65(2), 231-243. doi: 10.1007/s40732-014-0103-2

Gil-Luciano, B., Ruiz, F. J., Valdivia-Salas, S., \& Suárez-Falcón, J. C. (2017). Promoting psychological flexibility on tolerance tasks: Framing behavior through deictic/hierarchical relations and specifying augmental functions. The
Psychological Record, 67(1), 1-9. doi: 10.1007/ s40732-016-0200-5

Gomes, C. T., Perez, W. F., de Almeida, J. H., Ribeiro, A., de Rose, J. C., \& Barnes-Holmes, D. (2019). Assessing a derived transformation of functions using the implicit relational assessment procedure under three motivative conditions. The Psychological Record, 69(4), 487-497.

Guilhardi, H. J. (2002). Problemas e perspectivas na análise aplicada do comportamento: o caso da clínica. Apresentação no VI LABEX (Encontro de Pesquisadores do Laboratório de Psicologia Experimental da PUC-SP). São Paulo.

Hayes, S. C., Barnes-Holmes, D., \& Roche, B. (2001). Relational frame theory: A post-Skinnerian account of human language and cognition. New York: Kluwer Academic Publishers.

Hayes, S. C., Barnes-Holmes, D., \& Wilson, K. G. (2012b). Contextual behavioral science: Creating a science more adequate to the challenge of the human condition. Journal of Contextual Behavioral Science, 1(1-2), 1-16. doi: 10.1016/j.jcbs.2012.09.004

Hayes, S. C., Strosahl, K. D., \& Wilson, K. G. (2012a). Acceptance and commitment therapy: The process and practice of mindful change (2nd ed.). New York, NY: Guilford Press.

Hopkins, B. L., Cole, B. L., \& Mason, T. L. (1998). A critique of the usefulness of inferential statistics in applied behavior analysis. Behavior Analyst, 21(1), 125-137. doi: /10.1007/BF03392787

Johnston, J. M., \& Pennypacker, H. S. (2009). Strategies and tactics of behavioral research (3rd ed.). Routledge.

Kazdin, C. M. (1978). History of behavior modification: Experimental foundations of contemporary research. Baltimore: University Park Press.

Keogh, E., Bond, F. W., Hanmer, R., \& Tilston, J. (2005). Comparing acceptance and control-based coping instructions on the cold-pressor pain experiences of healthy men and women. European Journal of Pain, 9, 591-598. doi: 10.1016/j.ejpain.2004.12.005

Kishita, N., Muto, T., Ohtsuki, T., \& Barnes-Holmes D. (2014). Measuring the effect of cognitive defusion using the Implicit Relational Assessment Procedure: An experimental analysis with a highly socially anxious sample. Journal of 
Contextual Behavioral Science, 3(1), 8-15. doi: 10.1016/j.jcbs.2013.12.001

Levin, M. E., \& Villatte, M. (2016). The Role of Experimental Psychopathology and Laboratory-Based Intervention Studies in Contextual Behavioral Science. Em R. D. Zettle, S. C. Hayes, D. Barnes- Holmes, \& A. Biglan (Eds.), The Wiley handbook of contextual behavioral science (pp. 347-364). Hoboken, NJ: Wiley \& Sons.

Levin, M. E., Twohig, M. P., \& Smith, B. M. (2016). Contextual Behavioral Science: An overview. Em R. D. Zettle, S. C. Hayes, D. BarnesHolmes, \& A. Biglan (Eds.), The Wiley handbook of contextual behavioral science (pp. 17-36). Hoboken, NJ: Wiley \& Sons.

López-López, J. C., \& Luciano, C. (2017). An experimental analysis of defusion interactions based on deictic and hierarchical framings and their impact on cognitive performance. The Psychological Record, 67(4), 485-497. doi: 10.1007/s40732-017-0250-3

Luciano, C., Valdivia-Salas, S., Ruiz, F. J., RodríguezValverde, M., Barnes-Holmes, D., Dougher, M. J., ... \& Gutierrez-Martínez, O. (2014). Effects of an acceptance/defusion intervention on experimentally induced generalized avoidance: A laboratory demonstration. Journal of the Experimental Analysis of Behavior, 101(1), 94111. doi: $10.1002 /$ jeab. 68

Masuda, A., Hayes, S. C., Sackett, C. F., \& Twohig, M. P. (2004). Cognitive defusion and self-relevant negative thoughts: Examining the impact of a ninety year old technique. Behaviour Research and Therapy, 42(4), 477-485. doi: :10.1016/j.brat.2003.10.008

Matos, M. A. (1990). Controle experimental e controle estatístico: a filosofia do caso único na pesquisa comportamental. Ciência Cultura (Säo Paulo), 42(8), 585-592.

McMullen, J., Barnes-Holmes, D., BarnesHolmes, Y., Stewart, I., \& Cochrane, A. (2007) Acceptance versus distraction: Brief instructions, metaphors, and exercises in increasing tolerance for self-delivered electric shocks. Behaviour Research and Therapy, 46, 122-129. doi: 10.1016/j.brat.2007.09.002
Michael, J. (1980) Flight from behavior analysis presidential address ABA 1980. The Behavior Analyst, 3, 1-22. doi: 10.1007/BF03391838

Perez, W. F., Fidalgo, A. P., Kovac, R., \& Nico, Y. C. (2015). The transfer of Cfunc contextual control through equivalence relations. Journal of the Experimental Analysis of Behavior, 103(3), 511-523. doi:10.1002/jeab.150

Perez, W. F., Nico, Y. C., Kovac, R., Fidalgo, A. P., \& Leonardi, J. L. (2013). Introdução à Teoria das Molduras Relacionais (Relational Frame Theory): principais conceitos, achados experimentais e possibilidades de aplicação. Perspectivas em Análise do Comportamento, 04(1), 32-50.

Roche, B. T., Kanter, J. W., Brown, K. R., Simon, D., \& Fogarty, C. C. (2008). A comparison of "direct" versus "derived" extinction of avoidance responding. The Psychological Record, 58(3), 443-463. doi: 10.1007/BF03395628

Roche, B., Barnes-Holmes, D., Smeets, P. M., Barnes-Holmes, Y., \& McGeady, S. (2000). Contextual control over the derived transformation of discriminative and sexual arousal functions. The Psychological Record, 50,267-29. doi: 10.1007/BF03395356

Sampaio, A. A. S., De Azevedo, F. H. B., Cardoso, L. R. D., De Lima, C., Pereira, M. B. R., \& Andery, M. A. P. A. (2008). Uma introdução aos delineamentos experimentais de sujeito único. Interação Em Psicologia, 12(1), 151-164. doi: 10.5380/psi.v12i 1.9537

Sidman, M. (1960). Tactics of scientific research: Evaluating experimental data in psychology. Oxford, England: Basic Book.

Skinner B. F. (1966). What is the experimental analysis of behavior?. Journal of the Experimental Analysis of Behavior, 9(3), 213218. doi:10.1901/jeab.1966.9-213

Skinner, B. F. (1972). The operational analysis of psychological terms. Em B.F. Skinner, Cumulative record (pp.370-385). Appleton-Century-Crofts. Originalmente publicado em 1945.

Skinner, B. F. (2003). Ciência e comportamento humano (Vol. 10). São Paulo: Martins Fontes. Originalmente publicado em 1953.

Velasco, S. M., Garcia-Mijares, M., \& Tomanari, G. Y. (2010). Fundamentos metodológicos da 
pesquisa em análise experimental do comportamento. Revista Psicologia em Pesquisa, 4(2), 150-155.

Zettle, R. D. (2011). The evolution of a contextual approach to therapy: From comprehensive distancing to ACT. International Journal of Behavioral Consultation and Therapy, 7(1), 76-82. doi:10.1037/h0100929

Zettle, R. D., \& Hayes, S. C. (1986). Dysfunctional control by client verbal behavior: The context of reason-giving. The Analysis of Verbal Behavior, 4(1), 30-38. doi: 10.1007/bf03392813

\section{Informações do Artigo}

Histórico do artigo:

Submetido em: 26/11/2020

Primeira decisão editorial: 13/04/2021

Aceito em: 02/08/2021

Editor: William F. Perez 